\title{
Advances in model order reduction for fluid-structure interaction problems
}

\author{
Gianluigi Rozza*, Monica Nonino*, Francesco Ballarin*, andEfthymiosKaratzas ${ }^{\dagger}$ \\ * SISSA, mathematics area, mathLab, Via Bonomea 265, 34136 Trieste,Italy \\ e-mail:grozza@sissa.it, web page: http://people.sissa.it/grozza \\ ${ }^{\dagger}$ NationalTechnicalUniversity of Athens, 15780, Zografou, Greece
}

\begin{abstract}
Theaim of thisworkis to presentadvances and resultsontheapplication of modelorderreduction to thefield of fluid-structureinteraction (FSI) problems. Wedealwith a transportdominated FSI problem. Thesolutionmanifold has thus a slowlydecayingKolmogorov n-width, and thisdecreasestheeffectiveness of thereducedbasismethodbyincreasingthenumber of basisfunctionsnecessary to build a goodapproximationspace. Wepresent a preprocessingprocedure, carriedout in the offline phase, thatallows to decreasetheKolmogorov n-width of ourproblem. Wepresentsresultsthatshowthecomparison of the performances of theclassical offline stage and the new offline stage of thereducedbasismethod, in three test cases: a time dependent CFD problem (parametric and non-parametric), and a non-parametric time dependent FSI problem. Thisworkiscarriedout in collaborationwith Prof. Y. Maday. Thenwefocuson a partitionedapproach to FSI problems. Weimplementanalgorithmthatisbasedon a semiimplicitcouplingschemewithRobintypeboundaryconditions, takingintoaccountbothan ALEformulation and a geometricalparametrization of thedomain. Finally, weshowresultsconcerningtheapplication of CutFEM to FSI problems: from Stokes to Navier-Stokes and finallyconsidering a fullycoupledmultiphysicsproblem.
\end{abstract}

\section{REFERENCES}

[1] Nonino M., Ballarin F., Rozza G. and Maday Y., OvercomingslowlydecayingKolmogorov n-widthbytransportmaps: application to modelorderreduction of fluid dynamics and fluid-structureinteractionproblems, preprint, arXiv, 1911.06598, 2019.

[2] Ballarin F. and Rozza G., Galerkinmonolithicreducedordermodelsforparametrizedfluidstructureinteractionproblems, Int. J. Num. Meth. Fluids, (2016), 82:1010-1034.

[3] Ballarin F., Rozza G. and Maday Y., Reduced-ordersemi-implicitschemesfor fluidstructureinteractionproblems, ModelReduction of ParametrizedSystems, MS\&A, (2017), 17: $149-167$.

[4] Cagniart N., Maday Y. and Stamm B., ModelOrderReductionforProblemswithLargeConvectionEffects, Contributions to PartialDifferentialEquations and Applications, 2019, 131-150. 